\title{
LA CERTIFICACIÓN DE COMPETENCIAS LABORALES EN LACALIDAD DE LOS SERVICIOS: PERCEPCIÓN DE LOS TRABAJADORES DE LAS \\ EMPRESAS DE ALOJAMIENTO
}

\author{
Mirko Vera Campos \\ Instituto de Turismo, Universidad Austral de Chile \\ mirkovera@uach.cl \\ Natalia Albornoz Candía \\ Escuela de Turismo, Universidad Austral de Chile \\ natalia.albornoz.c@gmail.com \\ Constanza Rosas Opazo \\ Escuela de Turismo, Universidad Austral de Chile \\ constanza.rosas.opazo@gmail.com \\ Gloria Sanhueza Fernández \\ Escuela de Turismo, Universidad Austral de Chile \\ glo.sanhueza@gmail.com
}

\section{RESUMEN}

La presente investigación proporciona antecedentes sobre la percepción de los trabajadores de la industria de la hospitalidad respecto al programa de certificación de competencias laborales de Chile para la calidad de los servicios de alojamiento, ya que no existe suficiente evidencia empírica que permita sostener que la certificación de competencias de los trabajadores de la industria de la hospitalidad genere un incremento en la calidad del servicio. La implantación del Sistema Nacional de Competencias surge en Chile en el 2008 bajo el alero de la ley 20.267 e implementado por ChileValora. El sistema chileno busca reconocer formalmente las competencias laborales de las personas, ya que éstas son un componente fundamental en la productividad y competitividad de las empresas chilenas y, por consiguiente del país. Por otra parte, la literatura actual indica que para la industria de la hospitalidad la calidad del servicio resulta ser un factor fundamental, donde las personas conforman uno de los recursos más importantes para alcanzar la calidad en el servicio. Este estudio consideró la recopilación de datos mediante la aplicación de una encuesta a una muestra de 100 recepcionistas de establecimientos de alojamiento de la ciudad de Valdivia. Los resultados de la investigación demuestran que la certificación de competencias laborales es muy importante para la calidad del servicio según la precepción de los entrevistados, cuyo núcleo y/o esencia es el reconocimiento de la habilidad de los trabajadores en un área especifica de desempeño.

Palabras claves: Competencias Laborales, Certificación de Competencias, Hospitalidad, Calidad de Servicio, Recepcionistas. 


\title{
THE EMPLOYEES' PERCEPTION OF THE HOSPITALITY INDUSTRY RELATE TOTHE WOR KFORCE SKILLS CERTIFICATION PROGRAM OF CHILE FOR THE SERVICE QUALITY IN THE LODGING INDUSTRY
}

\author{
Mirko Vera Campos \\ Instituto de Turismo, Universidad Austral de Chile \\ mirkovera@uach.cl \\ Natalia Albornoz Candía \\ Escuela de Turismo, Universidad Austral de Chile \\ natalia.albornoz.c@gmail.com \\ Constanza Rosas Opazo \\ Escuela de Turismo, Universidad Austral de Chile \\ constanza.rosas.opazo@gmail.com \\ Gloria Sanhueza Fernández \\ Escuela de Turismo, Universidad Austral de Chile \\ glo.sanhueza@gmail.com
}

\begin{abstract}
This research provides information about the employees' perception of the hospitality industry related to a workforce skills certification program in Chile for the service quality in the lodging industry. The workforce skills certification emerges as an important contribution to the development of the Chilean companies and businesses. However, in the hospitality industry there is not enough empirical evidence to demonstrate that the skills certification of the employees increases the service quality. The implementation of the workforce skills certification national system arises in Chile in 2008 based on the Act 20267. Surprisingly, little attention has been paid to the importance of workforce skills certification system for the quality of service, particularly in the hospitality industry. This investigation collected data using a survey technique which was applied to a sample size of 100 receptionists in the lodging industry of Valdivia city. On the whole, the research's result shows that the workforce skills certification is very important for the service quality. These results are important in order to validate a program where the essential core is to recognize the skills of the employees in a particular occupation.
\end{abstract}

Key words: Workforce Skills, Skills Certification Program, Hospitality, Service Quality, Employee perception. 


\section{INTRODUCCIÓN}

El presente trabajo de investigación pretende aportar antecedentes sobre qué tan significativo es el reconocimiento de las competencias laborales para la calidad de los servicios en la industria del alojamiento, desde la perspectiva del trabajador. Este estudio plantea que el reconocimiento y certificación de las competencias del trabajador de la industria del alojamiento debería repercutir de forma directa en la calidad de los servicios. Sin embargo, no existe evidencia suficiente que sostenga que los procesos de evaluación, certificación y reconocimiento de las competencias laborales del trabajador de la industria del alojamiento incremente la calidad en la entrega del servicio. Por tal motivo, surge una cuestión obvia, ¿en qué medida el reconocimiento y certificación de las competencias laborales impactan en la calidad de los servicios según la percepción del personal la industria del alojamiento? Los antecedentes empíricos descritos en los siguientes artículo, y vacíos en la literatura en esta materia, aportan una mejor compresión del esta problemática.

Los trabajadores son un factor fundamental del sistema productivo de Chile. Sin embargo, según ChileValora (2014), en Chile existen cerca de 3,5 millones de personas que se desempeñan en el mercado del trabajo sin disponer de un título, ni reconocimiento formal que certifique su profesionalismo, conocimiento y/o experiencia. De este modo, para abordar esta situación nace y se promulga en Chile la ley número 20.267 del Sistema Nacional de Certificación de Competencias Laborales con el objeto de llevar a cabo el reconocimiento formal de las competencias laborales de las personas. Esta ley establece la creación de una institucionalidad en esta materia, bajo el alero de lo que hoy se conoce como ChileValora, y de Organismos Sectoriales de Competencias Laborales, cada uno con atribuciones definidas que permiten alcanzar los objetivos que el sistema se ha trazado. El objetivo de ChileValora es el reconocimiento formal de las competencias laborales de las personas que poseen habilidades no reconocidas, independientemente de la forma en que hayan sido adquiridas y si tienen o no un título o grado académico; así como favorecer las oportunidades de aprendizaje continuo de las personas, su reconocimiento y valorización (ChileValora, 2014). Este objetivo, a simple vista, plantea un cometido social del sistema más que una orientación hacia la mejora procesos productivos, en cualquiera de sus dimensiones como calidad, productividad, eficiencia, competitividad, entre otras.

La certificación de competencias laborales que otorga ChileValora podría formar un elemento de gran importancia para la industria de la hospitalidad, dado que mediante este proceso se podría otorgar un mayor grado de tangibilidad a las operaciones en la entrega de un servicio. Esto es relevante cuando la participación de las personas en la producción de los servicios es un factor fundamental. La industria de la hospitalidad 
incluye una amplia gama de servicios asociados al sector de la hotelería, gastronomía, entretenciones, viajes y excursiones (Costa, 2014). Según el Servicio Nacional de Turismo (2014), en el año 2010 este sector contribuyó con un 3,23\% al PIB constituyendo una importante fuente de empleo. Sin embargo, no existen estudios en Chile sobre los impactos de los procesos de reconocimiento y certificación de las competencias laborales sobre los procesos productivos o prestación de servicios, particularmente en la industria de la hospitalidad. Por lo que esta problemática es incipiente, poco desarrollada y muy significativa desde la perspectiva teórica, así como también para las políticas de gestión empresarial; esencialmente en el sector de servicios donde el factor humano es crucial.

Por otra parte, una amplia bibliografía sostiene que para la industria de la hospitalidad y el turismo la calidad de los servicios depende de las competencias de las personas que participan de su producción y entrega (Garcia, 2003; Irigoin \& Vargas, 2002; Torres, Chagas, \& Araújo, 2012). Esto llevar a suponer que, existe una positiva relación entre las certificación de competencia y el desempeño de los servicios. Más aún, la calidad del servicio se ha convertido en un elemento estratégico que confiere una ventaja diferenciadora y perdurable en el tiempo (Garza, Badii, \& Abreu, 2008a). Esto es particularmente importante para las empresas de servicios como la industria de la hospitalidad dado que el servicio es el núcleo del negocio. Además, las empresas de la industria de la hospitalidad dependen de objetivos estratégicos que tienen directa relación con la mejora de la calidad de los servicios.

\section{MARCO CONCEPTUAL}

\section{Competencias Laborales}

En la actualidad, el logro de los objetivos organizacionales se hace cada vez más complejo debido a que el sector empresarial experimenta continuos cambios (Escobar, 2005). Según Escobar (2005) estos cambios han sido influenciados por la globalización de la economía, la continua introducción de las nuevas tecnologías, la redefinición permanente de los procesos de producción y de administración en las organizaciones, entre otros. Por tanto, ante la complejidad de dichos escenarios, las organizaciones han centrado el logro de sus objetivos en el desarrollo del talento de las personas que conforman la empresa, dando así gran importancia a la certificación de competencias laborales para el logro de los objetivos organizacionales (Mertens, 1996).

Escobar (2005) plantea que muchas empresas han optado por la aplicación del Sistema de Competencias Laborales como método para potenciar al máximo las competencias de su personal. Esta estrategia empresarial se sustenta en que la certificación de competencias ayudará a crear y formar personas con grandes capacidades que lograrán desempeñarse adecuadamente consiguiendo cumplir las metas tanto personales como de 
la empresa (Barrera, Lorena, Calderari Ramírez, \& Álvarez, 2012).

El concepto de competencia nace en la década del 60, vinculado al movimiento educativo (OIT, 2014). Desde entonces, diversas definiciones se han desarrollado para clarificar y precisar el concepto de competencia laboral. En términos general, según la OCDE (2005) la competencia es la capacidad para responder exitosamente a una demanda compleja o llevar a cabo una a actividad o tarea, incluyendo las actitudes, valores, conocimientos y destrezas que hacen posible la acción efectiva. Desde la perspectiva del trabajo, según Bunk (1994, p. 9) "posee competencia profesional quien dispone de los conocimientos, destrezas y aptitudes necesarias para ejercer una profesión (capacidades profesionales), puede resolver problemas profesionales de forma autónoma y flexible (cualificación) y está capacitado para colaborar en su entorno profesional y en la organización del trabajo". En términos generales, una competencia es una capacidad, susceptible de ser medida, necesaria para realizar un trabajo eficazmente; es decir, para producir los resultados deseados por la organización (Vargas, 2006). La ley Chilena ${ }^{1}$ define las competencias laborales como "las actitudes, conocimientos, y destrezas necesarias para cumplir exitosamente las actividades que componen una función laboral, según estándares definidos por el sector productivo".

Al mismo tiempo, diversos modelo se han propuestos para implementar sistemas de certificación de competencias. Sin embargo, existen diferentes visiones e interpretación sobre la implementación de los modelos de competencias laborales. Algunos modelos de competencias se basan una visión conductista de las teoría del comportamiento, en las cuales las conductas de los individuos causan las acciones de un desempeño deseado (Zambrano, 2007). Otros se basan en las competencias como elementos personales alejados de las funciones específicas (Weinert, 2004). Por ejemplo, Delgado (2000) sostiene que las competencias individuales forman parte de un sistema holístico que contribuyen a las competencias organizacionales. Desde esta perspectiva, la suma y engranaje de las competencias individuales se pueden llegar a transformar en la ventaja competitiva de una organización (Guerrero, de los Ríos, Gómez, \& Guillén, 2010). También, diferentes son los modelos de competencia basados en el modelo cognitivo y motivacional: apoyada en el proceso de aprendizaje y desarrollo de las competencias (Foss, 2004). Según Escobar (2005) existen dos enfoques en los cuales se han basado los modelos de competencias. Por una parte, está el enfoque anglosajón el cual se centra en el contenido del puesto de trabajo, así como en su relación con la estrategia global de la organización. Por otra lado está el enfoque francés, el cual se centra más en el individuo y su finalidad es actuar como elemento de auditoría en torno a la capacidad individual del sujeto y el esfuerzo de la organización por mantener su fuerza de trabajo en condiciones óptimas de empleabilidad. Ambos enfoques se centran en puesto de trabajo, donde las

1 Ley núm. 20.267 de 2008, Crea el Sistema Nacional de certificación de Competencias Laborales y Perfecciona el Estatuto de Capacitación y Empleo 
competencias labores focalizadas definen las competencias requeridas para tener un desempeño eficiente. En este sentido, Fernández y Gámez (2005) sostienen que para el análisis del puesto de trabajo se emplea el modelo de competencias integrales el cual establece tres niveles. "Las competencias básicas son aquellas de índole formativas que requiere la persona para desempeñarse en cualquier actividad productiva. Las Genéricas como aquellos conocimientos y habilidades que están asociados al desarrollo de diversas áreas ocupacionales y las competencias específicas las cuales refieren a aquellas competencias asociadas a conocimientos y habilidades en ocupaciones específicas que son necesarias para la ejecución de una función productiva"(Luna \& Maldonado, p. 267). En síntesis, un modelo por competencia describe la combinación de conocimientos, habilidades y características necesarias para desempeñarse de forma eficiente en un rol dentro de una organización, sistema productivo y/o de servicios (Serpell \& Ferrada, 2011).

\section{Modelo Chileno de Certificación de Competencias laborales}

Según el Servicio Nacional de Capacitación y Empleo(SENCE, 2009) el modelo de competencias laborales desarrollado en Chile ha sido concebido como instrumento de fomento productivo, teniendo como centro en la valorización y sistematización del quehacer laboral de las personas. El Sistema Nacional de Certificación de Competencias Laborales se crea a partir de la promulgación de la Ley 20.267, la cual entró en vigencia en Chile en el año 2008. A pesar de que no existe claridad respecto a cuál son las bases teóricas del modelo chileno, se puede inferir que éste adopta un enfoque francés con fuerte énfasis en el reconocimiento de las habilidades adquiridas por un trabajador, por sobre la contribución de la competencia al desarrollo productivo. Por los cual, es claro que modelo chileno busca reconocer las habilidades, destrezas y aptitudes desarrollan las personas para desempeñar una actividad laboral. Esto está claramente especificado en la Ley 20.267, la cual expresamente señala que la certificación laboral cosiste en "el reconocimiento formal de las competencias laborales de las personas, independiente de la forma en que hayan sido adquiridas y de si tienen o no un título o grado académico otorgado por la enseñanza formal de conformidad las disposiciones de la Ley Orgánica Constitucional de Educación; así como favorecer las oportunidades de aprendizaje continuo de las personas, su reconocimiento y valorización.”(Ley 20.267, 2008). De acuerdo a lo especificado por la Ley, el modelo chileno de competencias laborales no tendría un enfoque de instrumento de fomento productivo como lo sostiene el SENCE, tampoco existe un compromiso táctico en dicha Ley que afirme que la evaluación, reconocimiento y certificación de las competencias asegure el desempeño eficiente de trabajadores en los lugares de trabajo. 
El modelo chileno de certificación de competencias laborales se basa en tres ejes estructurales (ver figura 1). La Comisión Nacional de Certificación de Competencias Laborales conocida como ChileValora, los Organismos Sectoriales de Competencias Laborales y, los Centros de Evaluación y Certificación. ChileValora es la institución responsable de la conducción estratégica y técnica del Sistema Nacional de Certificación de Competencias Laborales. ChileValora promueve la colaboración de los actores sindicales, gremiales y del estado, relacionados con los diferentes sectores productivos, para conocer en detalle las funciones de Chile Valora. Por otra parte, los Organismos Sectoriales de Competencias Laborales (OSCL) son una instancia estratégica de coordinación y consenso, conformada por representantes de los trabajadores, empleadores y del Estado vinculados a un sector productivo. Su función es identificar los perfiles ocupacionales prioritarios, validarlos, velar por su vigencia, actualizarlos y solicitar a ChileValora su acreditación a través de los Centros de Evaluación y Certificación de competencias laborales.

Figura 1, Modelo chileno de certificación de competencias laborales

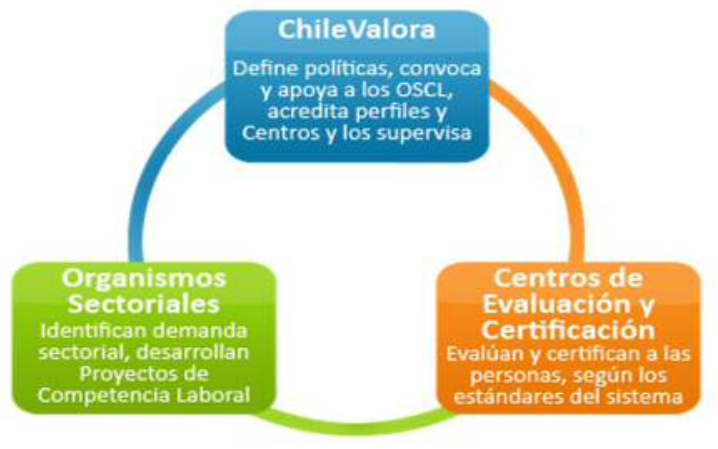

Fuente: ChileValora

Este proceso de evaluación se realiza través de los Centros de Evaluación y Certificación de Competencias Laborales debidamente acreditados por ChileValora (Cortés, 2013). Estos centros son entidades independientes que tienen la facultad para evaluar y certificar las competencias laborales de las y los trabajadores que lo soliciten, según los perfiles de competencias definidos por ChileValora. El reconocimiento de las competencias se materializa con la entrega de un certificado a cada trabajador que participa en el Proceso de Evaluación y Certificación de Competencias y que cumpla con los estándares de desempeño en relación con las competencias definidas por el sector productivo y de acuerdo al Catálogo de Competencias Laborales que administra ChileValora. Así, los procesos de evaluación permiten conocer el nivel de competencia de las personas respecto de un mínimo satisfactorio predefinido por un estándar. 
Dicho catálogo de competencias es un registro público que integra el almacenamiento organizado y oficial de los datos de cada uno de los perfiles ocupacionales acreditados. El perfil ocupacional es la agrupación de Unidades de Competencia Laboral (UCL) relevantes para una determinada área ocupacional u oficio. Las UCL son la descripción de los conocimientos, las habilidades y aptitudes que una persona debe tener para desempeñarse en distintas situaciones de trabajo en un puesto determinado.

\section{Calidad de los servicios}

Los estudios formales acerca de la calidad del servicio tienen su inicio con los trabajos de Parasuraman, Zeithaml, y Berry (1985). Las investigaciones sobre la calidad de los servicios han presentado una gran problemática debido a sus características de intangibilidad, heterogeneidad e inseparabilidad (Parasuraman et al, 1985). Por intangibilidad entendemos aquella cualidad de los servicios en la que no es fácil contarlos, medirlo, inventariarlos, y verificarlos o probarlos antes de entregarlos para asegurar su calidad. La heterogeneidad se refiere a que la mayoría de los servicios son variables, especialmente aquellos con gran participación de trabajo humano como los hoteles, esto quiere decir que a menudo su desempeño varía de cliente a cliente y de tiempo a tiempo, siendo difícil de garantizar similitud en la percepción de calidad. La inseparabilidad se refiere que la producción y el consumo ocurren simultáneamente, como consecuencia la calidad de los servicios solo es definida en la interacción del cliente y el personal (Escamilla Santamaría \& Duque Oliva, 2011; Meroño \& Bueno, 2014; Varo, 1994).

Desafortunadamente, la definición y medición de la calidad han resultado ser particularmente complejas de lograr en el ámbito de los servicios. Más aún, la literatura en este tema es muy amplia y no cuenta con una definición clara y universalmente aceptada respeto a la calidad del servicio. Según Horovitz (1997) se entiende por servicio como el conjunto de prestaciones que el cliente espera, además del producto o del servicio básico, como consecuencia del precio, la imagen y la reputación del mismo. Por otra parte, se puede afirmar que el concepto de calidad se refiere no solo a la calidad de los servicios entregados, sino también a la calidad de los procesos que se relacionan con dichos servicios (Garza, Badii, \& Abreu, 2008b; Oliva, 2005).

Camisón (1996) afirma que en una empresa de servicios, como las empresas turísticas, el principal recurso productivo son las personas que lo producen. De acuerdo a Camisón (1996) la calidad en el servicio es la principal fuente de las ventajas competitivas. Uriel, Monfort, Ferri, and Fernández de Guevara (2001) sostienen que cualquier empresa moderna reconoce la importancia que representar los recursos humanos en el desarrollo de la calidad de los servicios. Esta realidad es muy significativa en las empresas turísticas, donde el rol de los recursos humanos resulta fundamental en 
la producción y entrega del servicio. A raíz de ello, resulta interesante determinar de qué manera la certificación de competencias laborales influye en la calidad del servicio, y la producción del mismo servicio.

En relación a lo anterior, cabe señalar que existen dos posturas o corrientes que estudian la calidad de los servicios. Por un lado encontramos la escuela Nórdica, y por otro lado la Norteamericana (Oliva, 2005). El modelo nórdico liderado por Grönroos (1984) sostiene que existen dos dimensiones para determinar la calidad de servicio. La primera es la Dimensión Técnica (o también Diseño del Servicio) que se trata de valorar correctamente lo que esperan los clientes; y la segunda es la Dimensión Funcional, que se trata básicamente del cómo se ofrece el servicio. Por otra parte, la escuela Norteamericana se basa en el modelo SERVQUAL, el cual es una herramienta para la medición de la percepción sobre calidad del servicio. El modelo fue desarrollado por Parasuraman, Zeithaml, and Berry (1985) y ha experimentado continuas mejoras y revisiones. El modelo SERVQUAL se creó en principio para tratar de medir la desviación que hay entre las expectativas del cliente al hacer uso de un determinado servicio. El modelo tiene propósito mejorar la calidad de servicio ofrecido (Clemenza, Gotera, \& Rubén, 2010). El modelo SERVQUAL mide lo que el cliente espera de la organización que presta el servicio considerando cinco dimensiones básicas. Estas cinco dimensiones son:

- Confianza o Empatía en el servicio: Conocimiento del servicio prestado, cortesía de los empleados y su habilidad para transmitir confianza al cliente.

- Fiabilidad y cocimiento del servicio: Habilidad de prestar el servicio prometido tal como se ha prometido con error cero.

- Responsabilidad en la entrega del servicio: Esta dimensión mide la eficiencia y eficacia en la prestación del servicio. Con la eficiencia se consigue aprovechamiento de tiempo y materiales y la eficacia no es más que el cliente obtiene el servicio requerido

- Capacidad de respuesta: El deseo de ayudar y satisfacer las necesidades de los clientes de forma rápida y eficiente. Prestar el servicio de forma ágil.

- Elementos tangibles: Apariencia de las instalaciones equipos, empleados y materiales de comunicación como su distribución y facilidad de uso.

Según el modelo SERVQUAL la organización estará en condiciones de entregar un servicio de calidad cuando el personal en contacto alcanza las competencias laborales requeridas para el desempeño dimensiones específicas del modelo. De este modo, la calidad de los servicios estaría condicionada según el nivel de competencias 
que un trabajador pueda poseer. Sin embargo, no está del todo claro si el trabajador percibe que el reconocimiento de sus competencias impacta en la calidad servicio.

\section{Importancia de la certificación de competencias en la industria alojamiento}

En la industria de la alojamiento el perfil del personal de atención al cliente/ huésped, debe responder a competencias específicas. Estas competencias deben estar enfocadas para lograr la satisfacción del usuario de los servicios alojamiento. Por lo tanto, el prestador de servicio de alojamiento debe basar la calidad en las competencias de quienes participan en producción del servicio para incrementar el nivel de satisfacción de los usuarios. Sin embargo, la calidad de servicio en la industria alojamiento es difícil de medir debido a las propias características de los servicios (Santomá \& Costa, 2007). Los servicios de alojamiento constituyen una parte importante de la cadena de comercialización en los productos turísticos. Por tal motivo, el Gobierno de Chile desde hace un tiempo ha implementado el sello de calidad turística para garantizar calidad de los servicios entregados, particularmente en alojamientos turísticos. Actualmente, el Sistema de Calidad Turística Chileno cuenta con dieciséis normas técnicas en esta materia, que establecen requisitos mínimos con los que debe cumplir respecto de su organización, servicios, infraestructura y competencias laborales de las personas que allí trabajan. Su finalidad es brindar transparencia al mercado y otorgar una garantía de calidad del servicio a los clientes.

La significancia de las competencias laborales para la calidad de los servicios de alojamiento es reconocida por los expertos en investigación en la calidad de los servicios. La literatura señala que la relación entre el personal en contacto y el cliente es fundamental en la calidad de los servicios (descals, Eiglier, \& Langeard, 1989). "Cuando se definen las competencias, características y perfiles del equipo humano requerido, se debe tener en cuenta la capacidad de servicio al cliente de todas las personas que van a tener un nivel de visibilidad y una relación con el cliente" (Galindo, 2013, p. 49). Del mismo modo, la calidad y alcance del desempeño de las personas en funciones específicas dependen del nivel y tipo de competencias laborales que ha desarrollado en un momento determinado (Sanabria \& Pulido, 2012).

Según Santomá y Costa (2007) el desempeño de los empleados en términos de profesionalidad, capacidad de respuesta, trato cordial son determinantes de la calidad de los servicios de alojamiento. Es indudable que el personal es quien aporta la diferencia en la industria de alojamiento; esto lleva a pensar que la certificación de competencias parece ser una factor clave (Guerra Moreno \& Cardozo Sánchez, 2010). Por consiguiente, aun cuando se reconoce que el desarrollo de ciertas competencias resulta esencial para la calidad de los servicios en la industria de alojamiento, no existen estudios que demuestren que el reconocimiento y certificación de las mismas son 
relevantes o incrementan la calidad en la entrega de aquellos servicios, particularmente en la industria del alojamiento.

\section{METODOLOGÍA}

Las bases conceptuales de la presente investigación fueron proporcionadas en los apartados anteriores. En términos generales, esta investigación se centra el entender y reportar ¿En qué medida el reconocimiento y certificación de las competencias laborales impactan en la calidad de los servicios según la percepción de los trabajadores de la industria del alojamiento? Por lo cual, el estudio tiene como objetivo describir la relación existente entre la certificación de competencias laborales y la calidad del servicio en las empresas de alojamiento, como contexto del estudio se consideró la percepción de los recepcionistas de los establecimientos de alojamiento de la ciudad de Valdivia, Región de los Ríos de Chile.

La metodología se estructura según las recomendaciones proporcionadas por Monjes (2011). Según Monjes (2011) el proceso de investigación se construye de tres fases principales; base conceptual, planeación y diseño y finalmente analítica.

En cuanto a la fase de planeación y diseño; el diseño de la investigación es de tipo no experimental, por lo que estudio adopta un enfoque de tipo descriptivo con una orientación cuantitativa (Namakforoosh, 2000). En este estudio se recopilaron datos objetivos, precisos y sistemáticos mediante una encuesta aplicada a recepcionistas de empresas de alojamiento. De hecho, la esta investigación emplea técnicas de análisis de estadística descriptivas para explicar los resultados. La estadística descriptiva puede presentar información cuantitativa de forma simple y manejable, proporcionando resúmenes sencillos mediante gráficos para la visualización de los resultados (Muñoz, 2004).

La población o universo es el conjunto de sujetos que comparten características que permiten explicar la problemática del estudio. La población se encuentra conformada por los recepcionistas de establecimientos de alojamiento emplazados dentro la ciudad de Valdivia Región de los Ríos, Chile. Dado que no existe información específica respecto a la cantidad de recepcionistas que trabaja en los establecimientos de alojamientos, se tomó como referencia la cantidad de establecimientos de alojamientos que se encuentran registrados en el Servicio Nacional de Turismo de la Región de los Ríos (SERNATUR). Para poder cuantificar el universo y población se recurrió a la base de datos existente en la oficina de información turística de SERNATUR de la ciudad Valdivia. Para la muestra definitiva, se empleó el método de muestreo discrecional o por juicio correspondiente a un tipo de muestreo no probabilístico y en el cual los sujetos se seleccionan a base del conocimiento y juicio del investigador. Para el presente estudio los criterios de selección se utilizados fueron: 1) Accesibilidad al sujeto de estudio 2) El periodo de tiempo 
La Certificación de Competencias Laborales en la Calidad de los Servicios: Percepción de los Trabajadores de las Empresas de Alojamiento Gest. tur, $N^{\circ} 20$, julio - diciembre. 2013, pp 90-111 .
Mirco Vera

Natalia Albornoz

Constanza Rosas

Gloria Sanhueza

limitado para la ejecución de la investigación y 3) Los recursos puestos a disposición de la misma. Según los datos proporcionado por SERNATUR, existen en la región un total 373 unidades de establecimientos de alojamientos formalizados en la Región de los Ríos, a su vez en la ciudad de Valdivia existen un total de 134 establecimientos de alojamiento. Este estudio consideró un total de 100 recepcionistas del total de establecimientos de alojamiento de la ciudad de Valdivia.

Para la recolección de información se elaboró una encuesta que contiene tres partes: información general, indicadores de medición del estudio, y preguntas complementarias. En cuanto a las variables de medición de la calidad del servicio se consideraron las dimensiones propuesta por el modelo SERVQUAL. Para cada una de estas dimensiones se identificaron los indicadores o Ítems de medición, los cuales fueron tomados desde el perfil del recepcionista del Sistema Nacional de Certificación de Competencias de ChileValora. Por lo cual, el instrumento de investigación quedo constituido de 5 dimensiones y 34 Ítems de medición como se aprecia en la tabla 1 . Para la medición se elaboró una escala Likert de 1 a 5 , donde 1 es "Totalmente en desacuerdo" y 5 "Totalmente de acuerdo".

Tabla 1, Variables de medición

\begin{tabular}{|c|c|}
\hline Dimensiones & ftems \\
\hline $\begin{array}{l}\text { Confianza o Empatía } \\
\text { en el servicio }\end{array}$ & $\begin{array}{ll}\text { 1. } & \text { Realizo las actividades en los tiempos adecuados. } \\
\text { 2. } & \text { Me muestro atento, amable y empático con huéspedes y/o pasajeros que se presentan en el establecimiento hotelero. } \\
\text { 3. } & \text { Soy capaz de anticiparme a las necesidades de los huéspedes y/o pasajeros. } \\
\text { 4. } & \text { Resolver las necesidades de los huéspedes yio pasajeros es la actividad más importante que desempeño. } \\
\text { 5. } & \text { Entrego explicaciones claras e información fidedigna a los huéspedes y/o pasajeros además de un trato cordial. } \\
6 . & \text { Mantengo una actitud positiva y orientada a la calidad en la atención de pasajeros y/o huéspedes. } \\
7 . & \text { Aplico las politicas y protocolos de servicios de la empresa de manera cordial. }\end{array}$ \\
\hline $\begin{array}{l}\text { Fiabilidad } \\
\text { cocimiento } \\
\text { servicio }\end{array}$ & 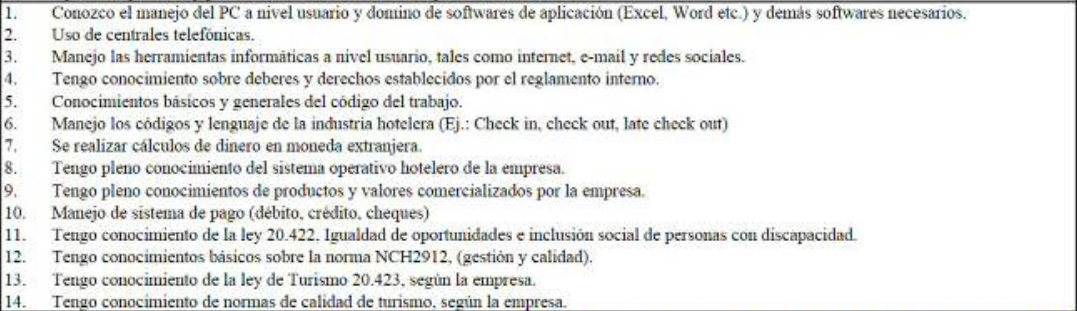 \\
\hline $\begin{array}{l}\text { Responsabilidad en la } \\
\text { entrega del servicio }\end{array}$ & $\begin{array}{l}\text { 1. Soy capaz de aceptar las diferencias del equipo teniendo una actitud tolerante ante modificaciones en las actividades que se encontraban } \\
\text { anteriormente planificadas. } \\
\text { 3. Soy capaz de cumplir con los plazos preestablecidos y con la calidad requerida para cl trabajo que realiza según indique la empresa. } \\
\text { 3. Soy capaz de identificar con claridad las actividades que requieren mayor dedicación y se redistribuir los tiempos para desarrollarlas } \\
\text { adecuadamente. }\end{array}$ \\
\hline $\begin{array}{l}\text { Capacidad de } \\
\text { respuesta en la entrega } \\
\text { del servicio }\end{array}$ & $\begin{array}{ll}\text { 1. } & \text { Soy capaz de enfrentar los conflictos con decisión y asertividad. } \\
\text { 2. } & \text { Comprendo el origen de uma situación conflictiva siendo persistente en la entrega de soluciones oportunas. } \\
\text { 3. } & \text { En la forma en que demuestra eficiencia y eficacia en la toma de decisiones bajo un contexto determinado. } \\
\text { 4. } & \text { Demuestro capacidad para comunicarme y expresarme de manera clara con quienes corresponda en las distintas situaciones que puedan } \\
\text { 5resentarse. } & \text { Identifico con claridad a quienes debo informar sobre situaciones o contextos conflictivos que puedan presentarse. }\end{array}$ \\
\hline $\begin{array}{l}\text { Tangibilidad del } \\
\text { servicio }\end{array}$ & $\begin{array}{ll}\text { 1. } & \text { La distribución de los espacios permite la entrega adecuada del servicio. } \\
\text { 2. } & \text { El equipamiento y disponible es apropiada a la demanda de los servicios. } \\
\text { 3. } & \text { La presentación de los empleados de la empresa es buena. } \\
\text { 4. } & \text { Las instalaciones fisicas son atractivas, modernas, limpias, organizadas y aptas para ofrecer un buen servicio. } \\
\text { 5. } & \text { Los elementos materiales (folletos, similares) son visualmente atractivos y claros. }\end{array}$ \\
\hline
\end{tabular}

Fuente: Elaboración propia 
La recolección de datos fue llevada a cabo en terreno por el grupo investigador, mediante la aplicación de una encuesta a 100 recepcionistas de establecimientos de alojamiento, durante el periodo de temporada estival, específicamente en el mes de febrero del año 2015.

\section{RESULTADOS Y DISCUSIÓN}

Los resultados obtenidos en la investigación siguen el mismo orden en que fueron recogidos y como se platearon en el diseño de la metodología. Específicamente, los resultados se presentan en tres partes: caracterización de la muestra en estudio, significancia de las competencias para la calidad de los servicios de alojamiento y grado de conocimiento del sistema certificación de competencia laboral. Los datos fueron tratados cuantitativamente para establecer la significancia de las variables consideradas en el estudio.

\section{Características de la muestra en estudio}

En este apartado se solicitó informaciones que permiten identificar y caracterizar al grupo en estudio, entre los datos solicitados están sexo y tipo de establecimiento en el que se encentran trabajando. Como se ha señalado en la metodología un total de 100 recepcionistas de establecimientos de alojamiento de la ciudad de Valdivia fueron encuestados, del cual el $64 \%$ de los encuestados es de sexo femenino, mientras que un $36 \%$ de sexo masculino. En cuanto al tamaño de los establecimientos, según el gráfico 1 los recepcionistas encuestados trabajan en establecimiento de alojamientos pequeños, según los estándares internacionales. Cerca del 50\% de ellos se concentra en establecimientos de entre 3 y 4 trabajadores. Esta no es una particularidad del área en estudio, sino más bien una característica de la industrial del alojamiento del país.

Gráfico 1, Tamaño establecimientos

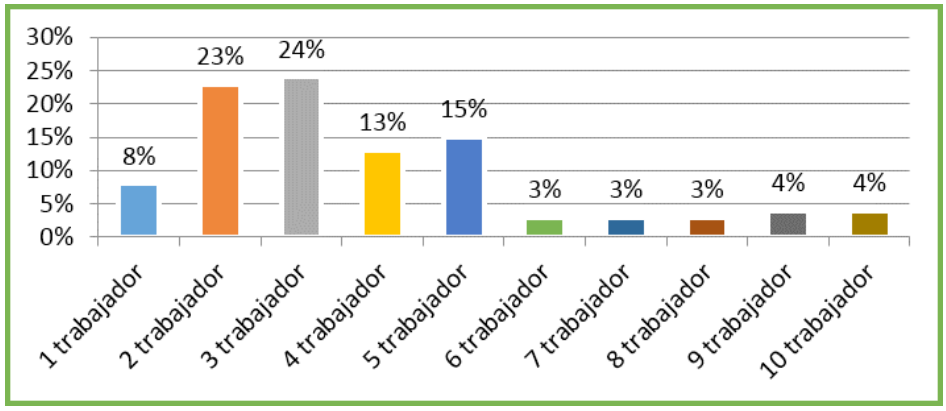

Fuente: Elaboración propia. 


\section{Significancia de las competencias para la calidad de los servicios de alojamiento}

En términos generales, los resultados demuestran que existe una relación directa entre la certificación de competencias y la calidad del servicio, como se aprecia en el gráfico número 2. Según la percepción de los encuestados, la certificación de las competencias son muy significativas en todas las dimensiones consideradas para medir de la calidad del servicio, tales como: Confianza y empatía en el servicio, fiabilidad y conocimiento del servicio, responsabilidad en la entrega del servicio, capacidad de respuesta del servicio y la tangibilidad. Tanto la media como la moda son altamente significativas, la moda es absoluta por los que la percepción de los encuestados es que todos los aspectos considerados en el perfil de competencias son relevantes para la calidad de los servicios de alojamiento.

Gráfico 2, Competencias laborales en la calidad del servicio de alojamiento

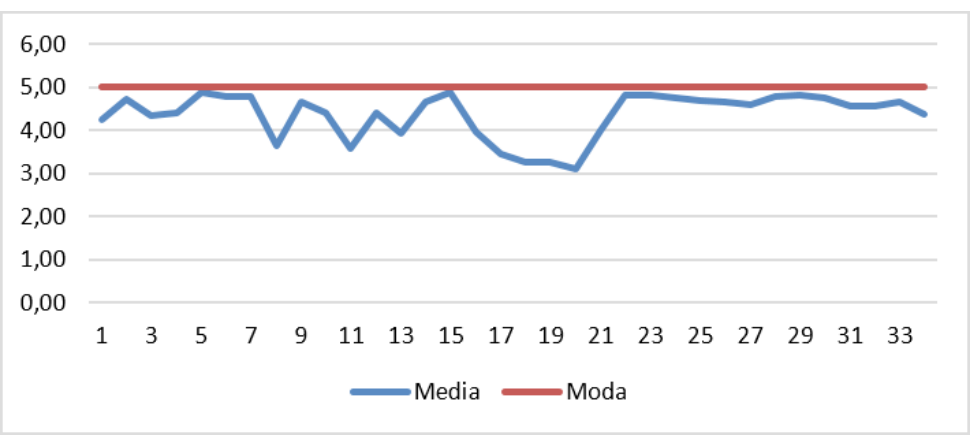

Fuente: Elaboración propia.

Concordantemente, la moda presenta un comportamiento similar en todas las dimensiones e ítems considerados. Sin embargo, se tuvo en cuenta el error estándar de medición que establece la precisión de la medida, el cual debería estar cercano a cero. Este permite precisar por que los ítems para analizar la dimensión de fiabilidad y cocimiento del servicio presenta un comportamiento menos uniforme, como se aprecia en el gráfico 2. En este sentido, según la percepción de los encuestados existen ciertas competencias que definidas en el perfil del Sistema Nacional de Certificación no necesariamente influyen en la calidad del servicio, los cuales tienen relación más con aspectos normativos de la calidad que la calidad en las operaciones de servicio. 
Los resultados muestran que existe una relación positiva entre las competencias laborales y la calidad del servicio. A continuación se presenta la descripción de los resultados por cada una de las dimensiones consideradas en el estudio y sus respectivos análisis.

Los 7 ítems considerados para evaluar la dimensión de empatía o confianza en el servicio son altamente significativos para la calidad del servicio. Según la percepción de los encuestados las competencias laborales relacionadas con el servicio prestado, cortesía de los empleados y actitud positiva y orientada a la calidad en la atención, así como su habilidad para transmitir confianza al cliente, son consideradas muy significativas para la calidad del servicio en terminos de confianza o empatía en el servicio, como se aprecia en el gráfico 3. Particularmente importante resulta la entrega de explicaciones claras e información fidedigna a los clientes.

Gráfico 3: Confianza o empatía del servicio

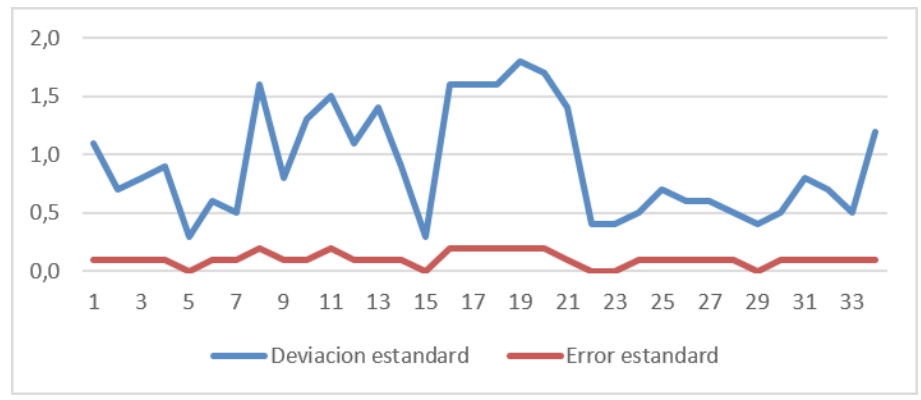

Fuente: Elaboración propia

Para medir la dimensión de fiabilidad y conocimiento del servicio se consideraron 14 ítems. Según la percepción de los encuestados existen competencias definidas en el Sistema Nacional de Certificación que no son significativas para la calidad del servicio, como se aprecia en el gráfico 4; esto puede deberse a que dichas competencias no se focalizan en evaluar en qué medida se cumple con prestar el servicio prometido con error cero. Las actuales competencias evalúan las variables normativas de los sistemas de calidad, más que a la calidad en las operaciones del servicio de alojamiento. 
Gráfico 4: Fiabilidad y conocimiento del servicio

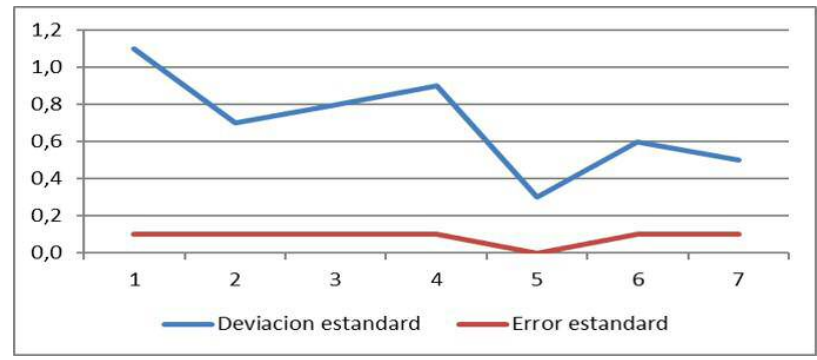

Fuente: Elaboración propia

Para medir la dimensión responsabilidad del servicio se consideraron 3 ítems que están contenidos en el Sistema Nacional de Certificación de Competencias, específicamente en el perfil del recepcionista. Como se aprecia en el gráfico 5, la percepción de los encuestados es que estas competencias son particularmente importantes para la calidad del servicio de alojamiento. Este tipo de competencias mide la capacidad individual en términos de eficiencia y eficacia en la entrega del servicio, debido a que considera aspectos de trabajo en equipo y cumplimiento de tareas.

Gráfico 5, Responsabilidad del servicio

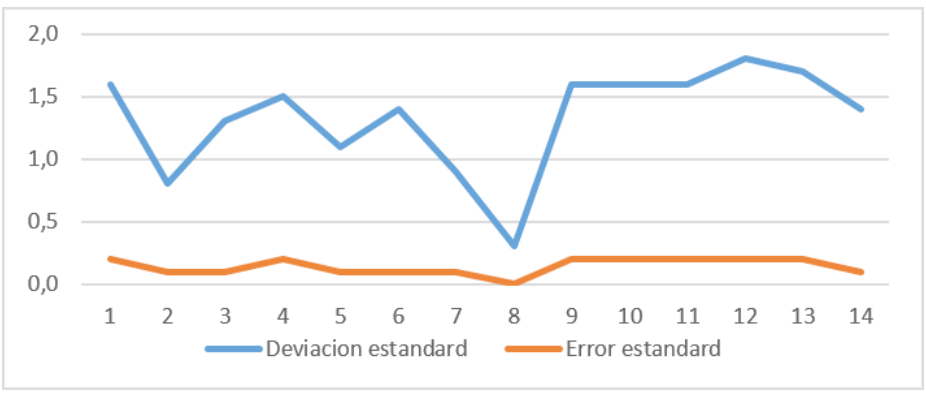

Fuente: Elaboración propia

Para la dimensión de la capacidad de respuesta en la entrega del servicio se consideraron 5 ítems, como se observa el grafico 6. En general, la percepción de los encuestados es que estas competencias influyen favorablemente en la calidad del servicio dado que expresan la capacidad de manejar situaciones conflictivas y toma de decisiones oportunas; así como, comunicar y expresar de manera clara para la entrega de información a los clientes. Esto repercute en el deseo de ayudar y satisfacer las 
necesidades de los clientes de forma rápida y eficiente.

Gráfico 6. Capacidad de respuesta en la entrega del servicio

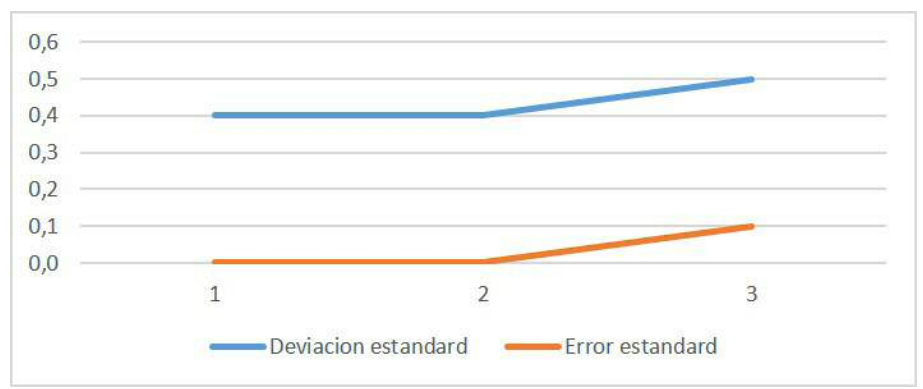

Fuente: Elaboración propia

Para la dimensión de tangibilidad de los servicios se consideraron 5 ítems, como se aprecia en el grafico 7. Si bien, los encuestados consideran que estas variables son importantes, no necesariamente son altamente significativas en la calidad de los servicios de alojamiento. Estas variables miden el contexto en el cual se entrega el servicio en términos de condiciones de las instalaciones y equipos, materiales, comunicación; así como, la distribución y facilidad de uso de los espacios.

Gráfico 7. Tangibilidad en el servicio

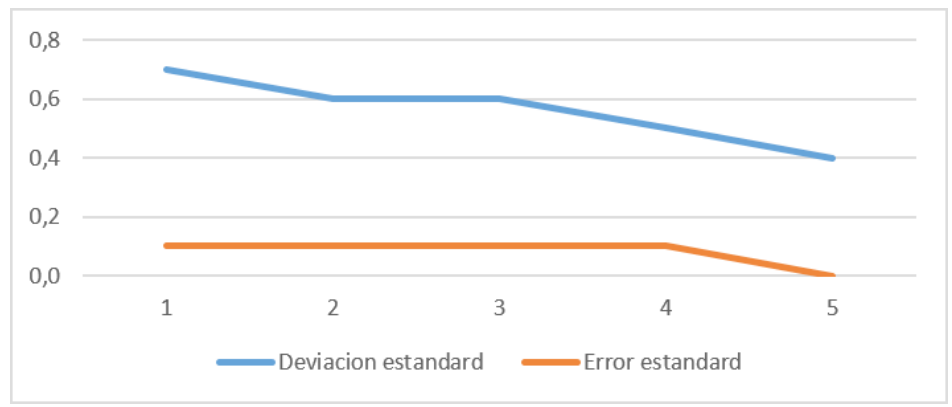

Fuente: Elaboración propia 


\section{Grado de conocimiento del Sistema Nacional Certificación de Competencia Laboral}

Esta sección describe brevemente el nivel de conocimiento que tiene el entrevistado respecto al Sistema Nacional Certificación de Competencia Laboral. En este sentido, del total de encuestados solamente el $27 \%$ declara conocer el sistema y ChileValora, mientras que un porcentaje significativo (73\%) que declara no tener mayores antecedentes del mismo. Por lo mismo, solo un $15 \%$ de los encuestados dice estar certificado bajo el Sistema Nacional de Certificación de Competencia Laboral, como se aprecia en el grafico 8 .

Gráfico 8. Nivel de certificación

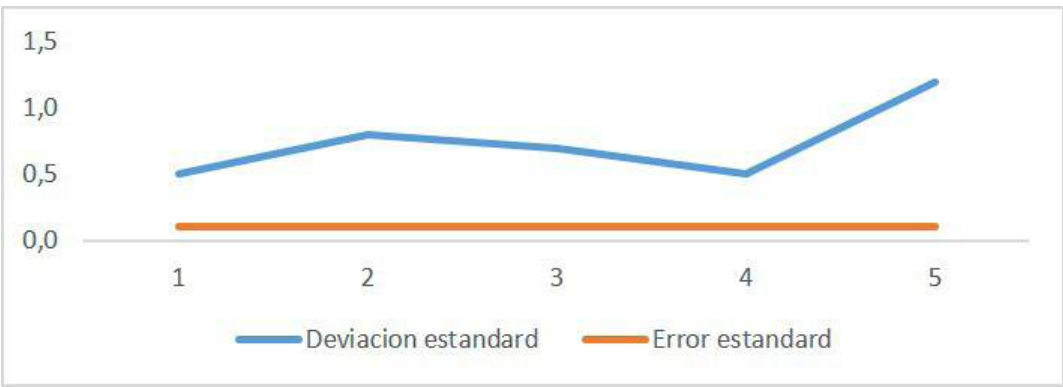

Fuente: Elaboración propia

\section{CONCLUSIONES}

Según los resultados de la presente investigación es posible sostener que la certificación de competencias laborales es un factor clave para la calidad de los servicios en la industria del alojamiento. En este sentido, debido a los cambios influenciados por la economía y el comportamiento de los consumidores el enfoque a la certificación de competencias laborales resulta clave para incrementar la percepción de calidad de los servicios de alojamiento. Las personas son un factor fundamental en la prestación del servicio de alojamiento, ya que ellas son las poseedoras de las competencias requeridas para ejercer influencia sobre la percepción de la calidad del servicio entregado. No obstante, la certificación de competencia no solo es un tema de reconocimiento de ciertas capacidades y destrezas de un trabador, sino también es un factor esencial en la competitividad de la industria del alojamiento. Existe una insipiente literatura que sugiere que las competencias laborales pueden influenciar significativamente la percepción sobre la calidad de los servicios. Sin embargo, se requieren estudios más acabados y extensos en esta materia. 
También, se pudo determinar que el Sistema Nacional de Certificación de Competencias Laborales de Chile, se caracteriza por perseguir objetivos más bien centrados en el trabajador, y no directamente relacionados con la productividad de las organizaciones o la mejora en la calidad de los servicios. Por lo que surgen diversas interrogantes respecto al impacto de la certificación de competencia en los procesos productivos, particularmente aquellos asociados a los servicios. Si el sistema se ha implementado para formalizar las capacidades no reconocidas de los trabajadores y con ello permitir la movilidad laboral, luego surgen interrogantes como ¿Cuáles son los impactos reales sobre el sistema productivo y la competitividad de la industria?

Si el sistema está orientado al trabajador los resultados tampoco son auspiciosos. Según los datos obtenidos más de la mitad de los recepcionistas encuestados no conocen la existencia del sistema de certificación de competencias laborales y Chile Valora. Esto evidencia que existe falta de difusión y mayor conocimiento por parte de los trabajadores del sistema. Esto podría explicar el bajo índice de trabajadores certificados en la industria de alojamiento. Esto es particularmente importante cuando los resultados de esta investigación demuestran que las competencias laborales son relevantes para mejorar la calidad de los servicios en la industria alojamiento.

Cabe mencionar que esta investigación es una primera aproximación para explicar un problema empírico de la industria de la hospitalidad y del turismo, donde la información es muy limitada. Si bien, algunos estudios se han llevado a cabo para explicar el impacto de la certificación de competencia en la productividad, la eficiencia, empleabilidad, entre otros problemáticas organizacionales, estos no son del todo concluyentes. También se evidenció una falta de investigación sobre el impacto de la certificación de competencias en la calidad de los servicios, particularmente en la industria del alojamiento. Esta investigación se llevó cabo considerando solo la percepción del trabajador, dado que el trabajador es el núcleo de los sistemas de certificación. Por lo que este estudio dejó fuera la percepción de los clientes; así como, la percepción de otros prestadores de servicios turísticos, tales como: restaurantes, agencias de viaje, tours operadores, entre otros. Sin embargo, estas limitaciones pueden considerarse como una oportunidad para profundizar y expandir el presente estudio. En definitiva, la certificación de competencias laborales supone una ventaja para el trabajador y para la organización. Para el trabajador el reconocimiento de sus competencias es un instrumento concreto de que le facilita la movilidad laboral. Para la organización es una oportunidad de fortalecer la calidad de los servicios por medio de la personas, certificando y reconociendo sus capacidades y habilidades. Finalmente, los resultados de esta investigación son acotados a los indicadores contemplados en las unidades de competencias definidos y validados en Chile, específicamente en el sector hotelero. 


\section{BIBLIOGRAFÍA}

Barrera, B., Lorena, M., Calderari Ramírez, S. M., \& Álvarez, J. E. (2012). Gestión por competencias: una opción para hacer las empresas más competitivas.

Bunk, G. (1994). La transmisión de las competencias en la formación y perfeccionamiento profesionales de la RFA. Revista europea de formación profesional(1), 8-14.

Camisón, C. (1996). La empresa turística: un análisis estratégico. Introducción a la economía del turismo en España, 217-245.

Costa, E. R. C. (2014). Comensalidad: La dádiva de la hospitalidad a través de la gastronomía. Estudios y perspectivas en turismo, 23(3), 505-525.

ChileValora. (2014). El capital humano es el corazón del mercado productivo Retrieved from http://www.chilevalora.cl/index.php/noticias-index/127-noticias-del2014/778-el-capital-humano-es-el-corazon-del-mercado-productivo website:

Clemenza, C., Gotera, A., \& Rubén, R. (2010). Calidad de los servicios prestados por el servicio autónomo municipal de administración tributaria. Revista Venezolana de Gerencia, 15(49).

Cortés, J. (2013). Manual para el Seguimiento y Supervisión a los Centros de Evaluación y Certificación de Competencias Laborales. In R. León (Ed.), (Vol. 01, pp. 22). Santiago de Chile ChileValora

Delgado, M. (2000). Modelos de gestión por competencias. 10. Retrieved from www. gestióndelconocimiento.com website:

Escamilla Santamaría, M. A., \& Duque Oliva, E. J. (2011). Revisión conceptual de la lealtad en servicios hoteleros. Criterio Libre, 9(14), 181-202.

Escobar, M. (2005). Las competencias laborales:; La estrategia laboral para la competitividad de las organizaciones? Estudios Gerenciales, 21(96), 31-55.

Fernández, J. T., \& Gámez, A. N. (2005). El desarrollo y la gestión de competencias profesionales: una mirada desde la formación. Revista Iberoamericana de Educación, 37(2), 4.

Foss, N. J. (2004). Cognition and motivation in the theory of the firm: interaction or" never the twain shall meet"? Journal des Economistes et des Etudes Humaines, 14(1).

Galindo, L. C. B. (2013). Hacia una correcta interpretación de 'servicio'. Vestigium. Revista Académica Universitaria, 2(2), 46-50.

Garcia, M. T. (2003). Comportamientos del personal de contacto y su influencia en la efectividad organizativa hotelera. Organización de Empresas y Comercialización. Universidad de Santiago de Compostela, España.

Garza, E., Badii, M., \& Abreu, J. (2008a). Mejoramiento de la c alidad de servicios mediante el modelo de las discrepancias entre las expectativas de los clientes y las percepciones de la empresa (Improvement of service quality through the discrepancy model between the expectations of the customers and the perceptions of the company). Daena: International Journal of Good 
Conscience, 3(1), 1-64.

Garza, E., Badii, M., \& Abreu, J. (2008b). Mejoramiento de la calidad de servicios mediante el modelo de las discrepancias entre las expectativas de los clientes y las percepciones de la empresa. Revista Daena (International Journal of Good Conscience), 3(1).

Grönroos, C. (1984). A service quality model and its marketing implications. European Journal of Marketing, 18(4), 36-44.

Guerra Moreno, K. M., \& Cardozo Sánchez, N. T. (2010). La gestión por competencias. Una herramienta para garantizar la calidad del servicio. Caso de estudio posadas turísticas del estado Táchira. Provincia(24), 31-51.

Guerrero, D., de los Ríos, I., Gómez, F., \& Guillén, J. (2010). Modelos internacionales de certificación de competencias profesionales: una caracterización de ocho modelos. Paper presented at the XIV INTERNATIONAL CONGRESS ON PROJECT ENGINEERING, Madrid, España

Horovitz, J. (1997). La calidad del servicio a la conquista del cliente\| Editorial Mc Graw Hill: España-Madrid.

Irigoin, M., \& Vargas, F. (2002). Competencia laboral: manual de conceptos, métodos y aplicaciones en el sector salud. In Cinterfor (Ed.), Cinterfor (pp. 252). Montevideo, Uruguay: Organización Internacional del Trabajo.

Luna, E. L., \& Maldonado, M. Y. C. (2006). Competencias laborales del trabajador social vistas desde el mercado laboral.

Meroño, C. P., \& Bueno, M. Á. B. (2014). Estrategias de Marketing Turístico/Touristic Marketing Strategies. Retos Turísticos, 13(1).

Mertens, L. (1996). Competencia laboral: sistemas, surgimiento y modelos: Cinterfor Montevideo.

Monjes, C. (2011). Metodología de Investigación qualitativa y quantitativa Universidad Surcolombiana, Colombia

Muñoz, D. R. (2004). Manual de estadística: Juan Carlos Martínez Coll.

Namakforoosh, M. N. (2000). Metodología de la investigación: Limusa.

OECD (2005) Definition and Selection of Key Competences (DeSeCo).Recuperado de https://www.oecd.org/pisa/35070367.pdf

OIT. (2014). La experiencia de ChileValora certificación de competencias laborales y diálogo social (Vol. ChileValora, pp. 109).

Oliva, E. (2005). Calidad del servicio y sus modelos de medición. Revista de ciencias administrativas y sociales, 15(25), 64-80.

Parasuraman, A., Zeithaml, V. A., \& Berry, L. L. (1985). A Conceptual Model of Service Quality and Its Implications for Future Research. Journal of marketing, 49(4), 41-50.

Sanabria, M. L. B., \& Pulido, L. H. O. (2012). Competencias en servicio al cliente y efectividad en solución de problemas. Sus características en el sector turismo. Estudios y perspectivas en turismo, 21(3), 646-662. 
Santomá, R., \& Costa, G. (2007). Calidad de servicio en la industria hotelera: revisión de la literatura. Revista de análisis turístico(3), 27-44.

SENCE. (2009). Modelo de Competencias Laborales Desarrollado en ChileNuevo Enfoque para la Capacitación Laboral. In S. N. d. C. y. Empleo (Ed.). Santiago-Chile SENCE.

Serpell, A., \& Ferrada, X. (2011). Modelo basado en competencias para formar, desarrollar y certificar supervisores de construcción. Revista Ingeniería de Construcción, 21(1), 43-56.

Servicio Nacional de Turismo, SERNATUR. (2014). Estrategia Nacional de Turismo de Chile 2012- 2020. Recuperado de http://portal.mma.gob.cl/wp-content/ uploads/2014/10/1_Estrategia-Nacional-de-Turismo-2012-2020.pdf

Torres, T. A., Chagas, M. M. d., \& Araújo, M. A. D. d. (2012). Competencias y habilidades necesarias de los gestores de hoteles de lujo y super lujo: Un estudio de caso en el Polo Turístico Via Costeira, Natal, Brasil. Estudios y perspectivas en turismo, 21(3), 604-620.

Uriel, E., Monfort, V., Ferri, J., \& Fernández de Guevara, J. (2001). El sector turístico en España. Caja de Ahorros del Mediterráneo, Valencia.

Vargas, F. (2006). Competencias en la formación y en la gestión de talento humano. Anales de la educación común, 2(Educación y trabajo), 147-163.

Varo, J. (1994). Gestión estratégica de la calidad en los servicios sanitarios: un modelo de gestión hospitalaria: Ediciones Díaz de Santos.

Weinert, F. (2004). Concepto de competencia: Una aclaración conceptual.: México: Fondo de Cultura Económica.

Zambrano, H. R. (2007). El paradigma de las competencias hacia la educación superior. Revista Facultad de Ciencias Económicas: Investigación y Reflexión, 15(1), 145-165. 